\title{
La bibliothèque publique comme lieu citoyen : variations sur un thème
}

\section{The Public Library as a Place for the Citizen: Variations on a Theme}

\section{Variaciones sobre la biblioteca pública como lugar ciudadano}

\section{Diane Mittermeyer}

Volume 50, numéro 4, octobre-décembre 2004

URI : https://id.erudit.org/iderudit/1030055ar

DOI : https://doi.org/10.7202/1030055ar

Aller au sommaire du numéro

Éditeur(s)

Association pour l'avancement des sciences et des techniques de la documentation (ASTED)

ISSN

0315-2340 (imprimé)

2291-8949 (numérique)

Découvrir la revue

Citer cet article

Mittermeyer, D. (2004). La bibliothèque publique comme lieu citoyen : variations sur un thème. Documentation et bibliothèques, 50(4), 265-272. https://doi.org/10.7202/1030055ar
Résumé de l'article

Ce texte est le résultat d'une réflexion effectuée dans le cadre du $31^{\mathrm{e}}$ congrès de l'ASTED, en octobre 2004. Le thème de la bibliothèque publique comme lieu citoyen soulève un certain questionnement : « La bibliothèque publique comme lieu citoyen : qu'est-ce à dire ? " Puisant tant aux sources de l'écrit, dont notamment le Manifeste de l'UNESCO sur la bibliothèque publique, qu'aux sources du quotidien : "Un dimanche après-midi à la bibliothèque ", cette réflexion tente d'illustrer la complexité du pas à franchir afin que nos bibliothèques deviennent des lieux citoyens à part entière.
Tous droits réservés $@$ Association pour l'avancement des sciences et des techniques de la documentation (ASTED), 2004
Ce document est protégé par la loi sur le droit d'auteur. L'utilisation des services d'Érudit (y compris la reproduction) est assujettie à sa politique d'utilisation que vous pouvez consulter en ligne.

https://apropos.erudit.org/fr/usagers/politique-dutilisation/ 


\title{
La bibliothèque publique comme lieu citoyen : variations sur un thème
}

\author{
DIANE MITTERMEYER \\ professeure agrégée \\ Graduate School of Library and Information Studies \\ Université McGill \\ Diane.mittermeyer@mcgill.ca
}

\section{RÉSUMÉ | ABSTRACTS | RESUMEN}

Ce texte est le résultat d'une réflexion effectuée dans le cadre du $31^{e}$ congrès de l'ASTED, en octobre 2004. Le thème de la bibliothèque publique comme lieu citoyen soulève un certain questionnement : «La bibliothèque publique comme lieu citoyen :qu'est-ce à dire?" Puisant tant aux sources de l'écrit, dont notamment le Manifeste de l'UNESCO sur la bibliothèque publique, qu'aux sources du quotidien : "Un dimanche après-midi à la bibliothèque», cette réflexion tente d'illustrer la complexité $d u$ pas à franchir afin que nos bibliothèques deviennent des lieux citoyens à part entière.

\section{The Public Library as a Place for the Citizen : Variations on a Theme}

This article is the result of an analysis that took place during the $31^{s t}$ ASTED conference held in October 2004. The theme, The Public Library as a Place for the Citizen, raised a number of issues. What exactly is meant by the public library as a place for the citizen? Drawing from a number of documents, namely the UNESCO Public Library Manifesto, as well as the daily publication Un dimanche après-midi à la bibliothèque, this analysis attempts to illustrate the complexity of the action needed in order for our public libraries to fully become places for the citizen.

\section{Variaciones sobre la biblioteca pública} como lugar ciudadano

Este texto es el resultado de una reflexión hecha para el $31^{\circ}$ Congreso de ASTED, en octubre de 2004. El tema de la biblioteca pública como lugar ciudadano plantea el problema de definir realmente qué significa «La biblioteca pública como lugar ciudadano». Con fuentes bibliográficas, como el Manifiesto de la UNESCO sobre la biblioteca pública, y artículos periodísticos como "Un dimanche après-midi à la bibliothèque» (Una tarde de domingo en la biblioteca), esta reflexión trata de ilustrar la complejidad del paso que se debe tomar para que nuestras bibliotecas se conviertan totalmente en «lugares ciudadanos».
1. Il faut préciser que ces définitions sont reproduites telles que présentées dans le dictionnaire consulté (incluant caractère gras, italique et lettres majuscules). a question étant posée : "La bibliothèque publique comme lieu citoyen : qu'est-ce à dire?», en début d'analyse, afin de mieux comprendre l'expression lieu citoyen, il a semblé utile de consulter le dictionnaire. Que la bibliothèque soit publique et qu'elle soit un lieu ne devraient pas être matière à un long débat, mais que cette bibliothèque soit un lieu citoyen n'est peut-être pas des plus évident. Aussi, en un premier temps, nous examinons quelques définitions.

\section{Définitions : un lieu citoyen}

Voyons tout d'abord la définition du mot lieu. Parmi les 11 définitions présentées dans le Robert, nous avons retenu les suivantes :

Lieu (N. M.) : 1. Portion déterminée de l'espace (considérée de façon générale et abstraite). = endroit, place. Être, se trouver dans un lieu.

3. Lieu public: lieu qui par destination admet le public (rue, jardin, mairie), ou auquel le public peut accéder (café, cinéma). ${ }^{1}$

(Le Robert illustré d'aujourd'hui, p. 839.)

Bien que le dictionnaire consulté préfère indiquer comme lieux publics la mairie, le café ou le cinéma à titre d'exemples, à notre avis, la bibliothèque aurait tout aussi bien pu illustrer cette définition. Donc, en tant que lieu (endroit, place) et qui plus est de lieu "auquel le public peut accéder», ce terme ne devrait pas faire l'objet d'une longue discussion.

En ce qui a trait à la définition du terme citoyen, notre ouvrage de référence ne présente que trois définitions, reproduites intégralement ci-après :

Citoyen (N) : 1. Individu considéré $d u$ point de vue de ses droits politiques. National d'un pays qui vit en république. = ressortissant. Un citoyen français et un sujet britannique. Accomplir son devoir de citoyen : voter.

-Citoyen du monde, qui met l'intérêt de l'humanité au-dessus du nationalisme.

2. sous la Révolution: Appellation pour monsieur, madame, mademoiselle.

3. Fam. Un drôle de citoyen: un individu bizarre.»

(Le Robert illustré d'aujourd'hui, p. 283-28) 
À la lumière de ces trois définitions, il convient de se demander : Quel sens peut-on attribuer à l'expression lieu citoyen dans un contexte de bibliothèque publique? On peut toujours considérer l'individu du point de vue de ses droits politiques ou encore comme citoyen du monde, mais une telle extrapolation ne contribue que peu à notre entendement. $\mathrm{Si}$, du point de vue politique, le citoyen peut être considéré comme un contribuable, c'est-à-dire un être versant des impôts sur ses revenus et participant ainsi à l'épanouissement de la bibliothèque publique, et qu'à son tour la bibliothèque contribue à l'épanouissement de nos citoyens en citoyens du monde, ces deux définitions ne nous éclairent pas vraiment sur ce qu'est un lieu citoyen.

Puisque la présente réflexion porte sur la bibliothèque publique comme lieu citoyen, nous devons opter pour la deuxième définition, soit celle apparue lors de la Révolution française: «une appellation pour monsieur, madame, mademoiselle». Ainsi, il est permis d'argumenter que la bibliothèque est effectivement un lieu public auquel le citoyen (monsieur, madame et mademoiselle) peut accéder. D'une telle définition s'ensuit que :

De la culture à l'information, du loisir à l'éducation, du réel au virtuel, [on peut] imaginer la bibliothèque comme lieu citoyen rassembleur où l'on s'active à partager sa culture, ses connaissances, ses loisirs...

(Extrait du Programme officiel, $31^{\mathrm{e}}$ congrès annuel de l'ASTED, p. 25)

Il convient donc de réfléchir :

... sur les possibilités de participation active de la bibliothèque dans la vie de la communauté. De la rencontre d'auteurs à la partie d'échecs, de la vente trottoir à un cours d'initiation à Internet, et pourquoi ne pas laisser la parole aux Ami(e)s... de la bibliothèque?

(Extrait du Programme officiel, $31^{\mathrm{e}}$ congrès annuel de l'ASTED, p. 25 )

\section{DÉFINITIONS : UNE BIBLIOTHĖQUE PUBLIQUE}

Avant de procéder, et afin de mieux situer le contexte de nos propos, examinons de plus près les définitions de deux autres termes : "publique» et «bibliothèque». Tout d'abord, dans notre contexte, quel sens doit-on attribuer au terme public (adjectif et nom)? La source présentée précédemment présente cinq définitions possibles ayant trait à l'adjectif et quatre définitions se rapportant au nom. De ces définitions, nous avons choisi les suivantes :
Public (ADJ.) : 1. Qui concerne le peuple dans son ensemble; relatif à la nation, à l'État. Relatif aux collectivités sociales juridiquement définies, à l'État. Les pouvoirs publics. La fonction publique. Service public. Lécole publique. = laïque.

2. Accessible, ouvert à tous. La voie publique. Jardin public. Réunion publique.

(Le Robert illustré d'aujourd'hui, p. 1162-1163.)

Public (n.m.) : 1. Les gens, la masse de la population. Le grand public: la population en général.

2. Ensemble des personnes que touche une cuvre (littéraire, artistique, musicale, un spectacle). Livrer son ouvrage au public.

(Le Robert illustré d'aujourd'hui, p.1163.)

À la lumière de ces définitions, l'expression lieu citoyen se précise. Par exemple, il est permis de supposer que monsieur, madame ou mademoiselle, représentant la masse de la population ou faisant partie du grand public, peut accéder à cette institution publique qu'est la bibliothèque, définie juridiquement en rapport avec la collectivité sociale qu'elle est appelée à desservir.

Bien que la plupart d'entre nous ayons une idée assez précise de ce qu'est une bibliothèque, si un citoyen (monsieur, madame ou mademoiselle) consultait Le Robert afin de se familiariser davantage avec cette institution publique, que trouverait-il ? Il y a trois interprétations possibles :

Bibliothèque (n.f.) :

1. Meuble permettant de ranger et de classer des livres. = aussi

rayonnage. Une bibliothèque vitrée.

2. Salle, édifice où sont classés des livres, pour la lecture ou pour le prêt. Bibliothèque de prêt, de consultation. Bibliothèque municipale. La bibliothèque d'Alexandrie. La bibliothèque de France.

3. Collection de livres. Un ouvrage de sa bibliothèque personnelle."

(Le Robert illustré d'aujourd'hui, p.156.)

Il est intéressant de constater qu'en 200o, date de l'édition du dictionnaire consulté, la bibliothèque ne soit définie que comme "salle, édifice où sont classés des livres, pour la lecture ou pour le prêt». Par contre, bien que la source consultée soit reconnue et, à notre avis, fort valable, il n'en demeure pas moins que celleci n'est qu'une source parmi tant d'autres. De nos jours, il y a fort à parier que pour ses besoins de définitions un citoyen consultera une source électronique comme 
par exemple Le Grand Dictionnaire terminologique de l'Office québécois de la langue française.

\section{DÉFINITIONS : LE GRAND DICTIONNAIRE TERMINOLOGIQUE}

Ainsi, au seul mot bibliothèque, on trouve 11 définitions dont une liée à l'édition, une autre à l'informatique, deux au mobilier et sept aux sciences de l'information. Voici, à titre d'exemple, la première définition du mot bibliothèque en lien avec les sciences de l'information :

BiBLIOTHÈQUe (n.f.) terme normalisé par un organisme international.

Définition: Organisme ou partie d'un organisme dont le but principal est de constituer des collections organisées de livres et de publications en série imprimés, ou tous autres documents graphiques ou audiovisuels, de les tenir à jour et de faciliter, grâce à un personnel spécialisé, l'utilisation de documents tels que ceux qui répondent à des besoins d'information, de recherche, d'éducation ou de loisir des usagers.

Office québécois de la langue française, 1991. http://www.granddictionnaire.com (consulté le 22 octobre, 2004)

S'il convient de noter l'approche plus générique de la présente définition, prenant en compte un ensemble de facteurs caractérisant la bibliothèque, comme par exemple, la diversité des documents, le personnel spécialisé et la diversité des besoins, à notre avis, une difficulté demeure : En quoi la bibliothèque publique est-elle un lieu citoyen, à part le fait que monsieur, madame et mademoiselle peuvent y accéder? Ou encore, selon les définitions présentées ci-dessus, ne conviendrait-il pas mieux de réfléchir davantage sur la bibliothèque dite publique que sur la bibliothèque dite lieu citoyen? Car, à notre avis, en ce qui a trait à la bibliothèque, qui dit «publique» dit «citoyens», tout comme qui dit «scolaire» dit «élèves et enseignants», ces adjectifs servant, somme toute, à délimiter les clientèles à desservir.

\section{LA BIBLIOTHÈQUE COMME LIEU PUBLIC}

Notre réflexion repose sur la définition de la bibliothèque en tant qu'institution publique, c'est-àdire accessible, ouverte à tous. Une institution dont l'accès et les services sont gratuits et ont pour but ultime de répondre aux besoins d'information, de loisir, de culture et d'éducation de tous les individus d'une collectivité sociale, celle-ci étant définie en lien avec les résidants (citoyens) d'une municipalité. Par contre, il faut préciser que la bibliothèque, en tant qu'institution publique, ne relève pas pour autant de l'administration municipale. En d'autres mots, la bibliothèque publique n'est pas nécessairement un service municipal, d'où notre appellation de bibliothèque publique et non de bibliothèque municipale.

Il est vrai que dans notre contexte québécois il n'est pas facile de se détacher de cette vision plutôt limitée de bibliothèque municipale définie principalement sous l'angle d'un service municipal dédié à la culture. Sur ce point, nous suggérons fortement la lecture d'un article de Silvie Delorme intitulé : «D'une institution culturelle à une institution démocratique. Passage obligé - L'ouverture citoyenne des bibliothèques publiques du Québec». Par exemple, si, depuis ses origines «La bibliothèque québécoise ne visait pas à former des citoyens responsables, mais plutôt à diriger les lectures du peuple» (Delorme, p. 43), en 1998, le ministère de la Culture et des Communications du Québec confirmait clairement sa vision culturelle de la bibliothèque publique dans une publication intitulée Le temps de lire, un art de vivre. La politique de la lecture et du livre. Selon Delorme (p. 44) :

Cette politique inscrit le rôle des bibliothèques dans le cadre de ce développement de la lecture et de la diffusion de la littérature... Or, cette politique fait peu de place au rôle civique de la bibliothèque et à l'importance de son apport au développement d'une société démocratique par le biais de l'information.

Parmi les nombreux effets limitatifs d'une telle vision, il appert que :

Le développement des bibliothèques publiques au Québec est freiné par le manque de sensibilisation des élus à l'importance du rôle de la bibliothèque, à la nécessité de l'information et de la formation.

(Delorme, p. 44)

\section{VARIATIONS SUR UN THÈME}

Nous avons choisi d'illustrer nos propos, d'une part, à partir du Manifeste de l'UNESCO sur la bibliothèque publique, et, d'autre part, de quelques expériences et faits vécus.

Le Manifeste de l'UNESCO sur la bibliothèque publique. Il va sans dire que ce choix n'est pas le fruit du hasard. Tout d'abord, ce manifeste pose, d'une manière succincte et précise (en cinq points), les bases d'une bibliothèque publique; deuxièmement, ce manifeste est susceptible de nous être familier puisque reconnu internationalement; et finalement, une version en langue française est facilement accessible 
à l'adresse : http://www.unesco.org/ webworld/libraries/manifestos/ libraman_fr.html

Soulignons d'abord l'importance qu'accorde le Manifeste à la connaissance, au savoir. Sous «Une porte ouverte à la connaissance», le rôle de la bibliothèque publique comme porte d'entrée du citoyen à la connaissance y est clairement précisé :

La liberté, la prospérité et le développement de la société et des individus sont des valeurs humaines fondamentales. Elles ne peuvent s'acquérir que dans la mesure où les citoyens sont en possession des informations qui leur permettent d'exercer leurs droits démocratiques et de jouer un rôle actif dans la société. Une participation créatrice et le développement de la démocratie dépendent aussi bien d'une éducation satisfaisante que d'un accès libre et illimité à la connaissance, la pensée, la culture et l'information.

La bibliothèque publique, porte locale d'accès à la connaissance, remplit les conditions fondamentales nécessaires à l'apprentissage à tous les âges de la vie, à la prise de décision en toute indépendance et au développement culturel des individus et des groupes sociaux. (http://www.unesco.org/webworld/libraries/manifestos/
libraman_fr.html) (consulté le 22 octobre, 2004)

Pour que la bibliothèque puisse bien jouer ce rôle de "porte locale d'accès à la connaissance», le Manifeste soutient qu'il faut «... tenir compte des missions-clés de la bibliothèque publique relativement à l'information, l'alphabétisation, l'éducation et la culture... » Aussi faut-il souligner l'aspect proactif de chacune des 12 missions-clés énoncées à la section Mission de la bibliothèque publique. La bibliothèque publique ne se contente plus de rendre accessibles la connaissance, le savoir, l'information. Le Manifeste de l'UNESCO lui confie la responsabilité de participer activement à la vie de la communauté. Elle a la responsabilité d'animer, de faire vivre ce lieu public au service des citoyens, par des actions concrètes répondant aux besoins spécifiques de sa communauté. Aussi dans les énoncés de missions-clés trouvons-nous une série de verbes d'action. Dans le passage qui suit, nous avons souligné ces verbes prônant la "participation active de la bibliothèque dans la vie de la communauté...» (Extrait du Programme officiel du $31^{\mathrm{e}}$ congrès annuel de l'ASTED, p.25) :

1. créer et renforcer l'habitude de lire chez les enfants dès leur plus jeune âge;

a) soutenir à la fois l'autoformation ainsi que l'enseignement conventionnel à tous les niveaux; b) fournir à chaque personne les moyens d'évoluer de manière créative;

c) stimuler l'imagination et la créativité des enfants et des jeunes;

d) développer le sens du patrimoine culturel, le goût des arts, des réalisations et des innovations scientifiques;

e) assurer l'accès' aux différentes formes d'expression culturelle des arts du spectacle;

f) développer le dialogue interculturel et favoriser la diversité culturelle;

g) soutenir la tradition orale;

h) assurer l'accès des citoyens aux informations de toutes catégories issues des collectivités locales;

i) fournir aux entreprises locales, aux associations et aux groupes d'intérêt les services d'information adéquats;

j) faciliter le développement des compétences de base pour utiliser l'information et l'informatique;

k) soutenir les activités et les programmes d'alphabétisation en faveur de toutes les classes d'âge, y participer et mettre en ouvre de telles activités, si nécessaire.

(http://www.unesco.org/webworld/libraries/manifestos/ libraman_fr.html) (consulté le 22 octobre, 2004)

L'orientation proactive de ces missions-clés invite nécessairement la bibliothèque publique à agir, à s'impliquer au sein de sa communauté. Désormais, l'accès à la connaissance, au savoir, à l'information, à la culture, à l'éducation ne se limite plus à la lecture d'un document, qu'il soit analogue ou numérique. Par exemple, "pour créer et renforcer l'habitude de lire chez les enfants", il faut davantage que développer une collection de documents et faciliter son accès. La bibliothèque publique se doit d'être un partenaire actif dans l'établissement de cette habitude de lecture chez l'enfant en offrant à ce citoyen en devenir un ensemble de programmes d'animation de la lecture. Elle a également la responsabilité de "stimuler l'imagination et la créativité des enfants et des jeunes». Il ne suffit pas de remplir à leur intention les rayons de la bibliothèque de documents, ni même de leur fournir l'accès à de beaux sites Web. Ici encore, une action directe par l'établissement de programmes offrant la possibilité de marier le document et la réalisation est de mise. Et que dire de la responsabilité de «développer le dialogue interculturel... » ou de «soutenir la tradition orale»? Autant de volets où l'accès à la connaissance et au savoir ne se limite pas au document, qu'il soit sur support papier, numérique ou autre. 
De ces 12 missions-clés, 2 nous interpellent particulièrement. Ces deux missions renforcent notre énoncé de départ - «La bibliothèque publique comme lieu citoyen"- puisqu'elles portent, pour ainsi dire, sur la responsabilité citoyenne de la bibliothèque :
Sous ce volet, bien que quelque peu restreinte, laction citoyenne de la bibliothèque est effectivement reconnue.

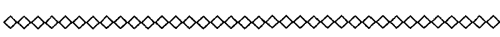

de la communauté avec lequel le bibliothécaire devrait apprendre à composer est le groupe des Ami(e)s de la bibliothèque. Complices inconditionnels de cette institution publique, les Ami(e)s regroupent un ensemble de citoyens-utilisateurs dont le mandat est précisément de représenter la communauté dans son ensemble. Selon Louise Jolicœur (p.127-128) : "L'absence de groupes d'amis serait un chaînon manquant expliquant une partie du retard historique qu'a pris le Québec en matière de bibliothèque publique.» services d'information adéquats.

Alors que la bibliothèque publique est accessible à tous et active auprès de tous (monsieur, madame et mademoiselle), sa participation active au sein de la communauté inclut, il va sans dire, sa responsabilité de canaliser et de rendre facilement accessible, par des services appropriés, l'information que l'on qualifie souvent d'«information communautaire». Sous ce volet, bien que quelque peu restreinte, l'action citoyenne de la bibliothèque est effectivement reconnue.

En dernier lieu, alors que, sous Financement, législation et réseaux, le Manifeste nous rappelle la nécessité de "... textes législatifs spécifiques... ", d'un soutien financier assuré «... par les autorités nationales et locales» ainsi que «... des normes de service appropriées", la dernière section, portant sur le Fonctionnement et [la] gestion, nous interpelle à nouveau. En bref :

La coopération avec les partenaires concernés - par exemple, des groupes d'utilisateurs et d'autres professionnels à l'échelon local, régional, national, de même qu'international-doit être assurée.

Le bibliothécaire est un intermédiaire actif entre les usagers et les ressources offertes. La formation professionnelle et continue $d u$ bibliothécaire est indispensable pour assurer des services adéquats.

(http://www.unesco.org/webworld/libraries/manifestos/ libraman_fr.html) (consulté le 22 octobre, 2004)

Dans le cadre de la présente réflexion, ces deux passages du Manifeste de l'UNESCO sur la bibliothèque publique indiquent clairement, à notre avis, que toutes les actions à accomplir afin de remplir les missionsclés de la bibliothèque ne peuvent se réaliser sans la participation et la coopération des divers partenaires concernés. Si le bibliothécaire se doit d'orchestrer les divers paliers d'une telle coopération, à l'échelon local, un partenaire actif et un précieux porte-parole

2. La bibliothèque visitée en ce dimanche après-midi du 17 octobre 2004 est la Bibliothèque Fraser-Hickson, située au 4855, avenue Kensington, à Montréal.

\section{Un lieu public au quotidien, qu'est-ce à dire?}

Devant la difficulté d'attribuer un sens précis à l'expression lieu citoyen et afin d'illustrer une approche jusqu'ici plutôt théorique, nous désirons soumettre à l'attention du lecteur quelques exemples de ce qu'est, à notre avis, une bibliothèque publique comme lieu citoyen, ou, plus simplement, ce qu'est une bibliothèque publique. Pour ce faire, nous avons choisi deux exemples. Le premier pourrait s'intituler : Un dimanche après-midi à la bibliothèque, alors que le deuxième pourrait s'intituler Une publication qui n'a pas son pareil.

\section{Un dimanche après-midi à la bibliothèque ${ }^{2}$}

Bien que la bibliothèque n'ait ouvert ses portes que depuis une demi-heure à peine, comme dans toutes les bibliothèques publiques un dimanche après-midi, la majorité des tables et des fauteuils de la grande salle de lecture sont déjà occupés. On peut y noter les activités usuelles : lecture du journal, consultation de documents de référence, prise de notes pour ce qui apparaît être un travail scolaire, lecture de détente - ici une revue de mode et là un roman. Parmi les autres activités usuelles, on peut noter la consultation du catalogue en ligne par quelques personnes; il y a aussi deux personnes qui attendent au Service de référence.

Scène de vie quotidienne sans plus, direz-vous, mais voilà... dans cette bibliothèque de quartier, un dimanche après-midi, les activités ne se limitent pas à la consultation du personnel ou de documents de référence, à la lecture de détente ou à la recherche de documents à emprunter. Dans un large corridor reliant l'espace desservant la clientèle adulte à celui desservant la clientèle parents-enfants-famille, on note une série de tables alignées près des fenêtres où déjà quelques personnes, attablées par groupes de deux, se concentrent d'un air sérieux. Le jeu d'échecs exige une telle concentration, n'est-ce pas! Oui mais... pas si l'on souhaite apprendre à jouer tout 
En un seul dimanche

en s'amusant... et c'est exactement cette activité qui débute à l'instant à la bibliothèque pour enfants, située à l'autre bout du corridor. Bien sûr, cet espace, beaucoup plus bruyant, possède son entrée propre et deux portes vitrées tentent avec un succès relatif d'isoler nos joueurs d'échecs du bruit que peuvent occasionner le rangement des poussettes et les cris d'enfants heureux de retrouver leur professeur. Car, tous les dimanches après-midi, on vient approfondir sa connaissance du jeu d'échecs en y jouant sous l'œil attentif et avec les judicieux conseils de professeurs bénévoles (parents, voisins et amis). Il ne reste que quelques mois avant le tournoi annuel, organisé conjointement par les professeurs bénévoles et le personnel de la bibliothèque et qui se tiendra dans l'auditorium de la bibliothèque. Bien entendu, avant de quitter cet espace famille, on note les activités usuelles, comme la lecture de livres d'images, ainsi que la consultation de la bibliothécaire, qui connaît tous les livres de la bibliothèque...

Adjacent à l'entrée de la bibliothèque desservant la clientèle famille, se trouve l'escalier menant à l'auditorium. En ce dimanche après-midi, une heure à peine après l'ouverture de la bibliothèque, on note une trentaine de personnes discutant, partageant, entre conjoints ou amis, leur évaluation des toiles faisant partie de la présente exposition. Depuis une semaine, un groupe local d'artistes peintres y exposent leurs toiles. À plusieurs d'entre eux, la bibliothèque offre ainsi la possibilité de rejoindre le public de leur quartier. Lorsque vient le moment d'une pause, on se donne rendez-vous au Café de la bibliothèque, qui a ouvert ses portes récemment. Situé de l'autre côté du corridor, où s'activent les joueurs d'échecs, en entrant au Café, on remarque deux jeunes dames et un jeune homme attablés devant un café et discutant de formules mathématiques, livres ouverts devant eux. À une autre table, se trouve un lecteur et, dernière lui, quelqu'un bouquine sur les rayons des livres usagés à vendre pour quelques dollars ou moins.

Finalement, ce jour-là, notre visite de la bibliothèque publique se termine par une rencontre des Ami(e)s de la bibliothèque, qui se tient au deuxième étage de l'édifice mais dans un local adjacent à la salle des ordinateurs, où se donnent normalement les cours d'introduction à la recherche sur Internet et le Web. Comme il n'y a pas de cours le dimanche, on note la présence de deux instructeurs bénévoles en grande discussion devant ce qui semble être un site Web, ou peut-être un logiciel... Notre point d'observation, qui se veut discret, ne permet pas d'être plus précis.

Voilà comment cette brève visite d'une bibliothèque de quartier nous a permis d'illustrer ce qu'est, à notre avis, une bibliothèque publique, ou, si l'on préfère, une bibliothèque publique comme lieu citoyen. En un seul dimanche après-midi, nous avons $\mathrm{pu}$ constater comment le citoyen (monsieur, madame et mademoiselle) avait accès à une foule d'activités en complément à la lecture et à la consultation de documents en bibliothèque ${ }^{3}$. Ce faisant, nous sommes désormais en mesure de témoigner, à l'aide d'exemples, de l'accomplissement d'un certain nombre des missions-clés énumérées dans le Manifeste de l'UNESCO sur la bibliothèque publique, qui appellent la bibliothèque à s'impliquer dans sa communauté. À titre d'exemples :

\section{3. fournir à chaque personne les moyens d'évoluer de manière créative (jouer aux échecs, discuter d'ouvres d'art, échanger des idées en prenant un café, ou en joignant un groupe communautaire, partenaire de la bibliothèque);}

stimuler l'imagination et la créativité des enfants et des jeunes (apprendre à jouer aux échecs);

développer le sens du patrimoine culturel, le goût des arts... (exposition de toiles);

développer le dialogue interculturel et favoriser la diversité culturelle (le groupe des Ami(e)s de la bibliothèque);

faciliter le développement des compétences de base pour utiliser l'information et l'informatique (des instructeurs bénévoles préparant un cours).

\section{Une publication qui n'a pas son pareil}

Pour ce second exemple visant à illustrer ce qu'est une bibliothèque publique ou une bibliothèque publique comme lieu citoyen, nous recommandons la lecture d'une revue exceptionnelle, @ the Library, publiée cinq fois l'an par la Bibliothèque publique de Regina, en Saskatchewan. À notre avis, cette publication passe avec brio le test de la communication d'une bibliothèque avec sa communauté. Bien qu'elle ne soit probablement pas la seule publication de ce genre à passer le test, nous l'avons choisie pour la qualité de sa présentation et de son contenu. Même si toutes les informations contenues dans cette publication se trouvent sur le site Web de la Bibliothèque, la version papier est susceptible de rejoindre davantage de citoyens.

\footnotetext{
3. Pour de plus amples détails sur des activités telles celles rapportées ici, nous invitons le lecteur à prendre connaissance de la publication Chapter $\mathcal{E}$ Verse produite par la Bibliothèque Fraser-Hickson.
} 
Le numéro consulté (vol. $\left.4, \mathrm{n}^{\circ} 4\right)$ comporte sept sections. La Politique de prêt (Circulation Policy) (p. 2) nous informe, entre autres, de l'utilisation de la carte de membre de la Bibliothèque. Celle-ci peut-être utilisée non seulement dans les diverses succursales de la Bibliothèque publique de Regina, mais également dans toutes les bibliothèques publiques de la Saskatchewan, compte tenu de certaines restrictions. Les autres sections nous informent des développements récents qu'a connus la Bibliothèque (Library News) (p. 4-7), la Programmation d'activités destinées à la clientèle adolescente (Teen Programs) (p.8), la Programmation d'activités destinées à la clientèle du troisième âge (Seniors' Programs) (p.9), les films à l'affiche au Cinéma de la Bibliothèque de Regina - Bibliothèque centrale (RPL Film Theatre) (p.10), les expositions de la Galerie d'art à la Bibliothèque centrale (Dunlop Art Gallery) (p.11) et, finalement, toute une série d'activités à l'affiche pour les deux prochains mois tant dans les huit succursales qu'à la Bibliothèque centrale (Around the Branches) (p.12-28). Le tout est complété, en pages 29, 30 et 31, par une section intitulée «At a Glance» présentant en résumé les diverses activités (date, horaire, sujet, endroit) du réseau des bibliothèques de Regina, du $1^{\mathrm{er}}$ septembre au 30 octobre 2004.

Ainsi, cette publication de format magazine, attrayante mais sans publicité, invite tous les citoyens (monsieur, madame et mademoiselle), ou, pourrait-on dire, le grand public, à participer à une foule d'activités, des plus diversifiées et toutes aussi intéressantes les unes que les autres. À titre d'exemples, nous avons sélectionné ce qui suit :

De la page Teen Programs :

Teens@ the Library-Meet approximately every other month and talk about what the Library can do for you! Members of the Teen Advisory Committee help decide what should be in our Young Adult collection and give advice on everything from programming to décor. For more information call Jessica at 7776095. (p. 8)

Rock ' $n$ ' Roll Over Dead - The Music Librarian has been found dead! Was it a heart attack or ... murder? Join your fellow detectives at Sherwood Village Library in solving the mystery. You must investigate the crime scene and carefully examine the evidence in order to solve the crime. Refreshments to follow. For detectives 10-18 years old. Space limited. Preregister by calling 777-6088. (p.8)

De la page Seniors' Programs :

Gambling and Seniors-An increasing number of seniors are gambling for fun and entertainment. However, gambling can become a serious problem. Dave Morgan,
Assistant Director of the Problem Gambling Community of the Canadian Mental Health Association, will discuss some of the factors that contribute to seniors' gambling problems and outline community resources that are available. Presented in co-operation with the Seniors' Healthy Living Program, Regina Qu'Appelle Health Region. (p. 9)

A Little of Everything-Armchair Travel Series-Join seasoned travelers as they transport you to locations around the world. Each Monday afternoon you will experience sights and sounds of a different locale. Coffee served. Co-sponsored with New Horizons Library Group. Pre-registration not required. (p.9)

Des pages Central Library:

Literacy Tutor Information Session-Are you interested in becoming a volunteer Literacy Tutor? Come to one of our information sessions to find out about the Library's Literacy Program and our Tutor Training Workshops for tutoring Adult Literacy Learners or English-as-a-Second-Language Learners. You will be introduced to our computer lab, literacy collection and tutor support services. Experienced tutors and literacy staff will be available to answer your questions and refreshments will be provided. Pre-register for one session only at 777-6009. (p.13)

Business Info@Lunch-Join our Business Reference Librarian, Jason Bird, in the Film Theatre for lunch hour sessions on the many resources available @ your library. Bring your brown bag lunch; coffee and cookies provided. For more information call 777-6200 or e-mail : jbird@reginalibrary.ca.October 7 : Business Books@Your Library; October14 : Getting the Competitive Edge: Business Databases@ the Library; October 21 : Business Reference Materials : Unearth its Treasures; October 28 : Government, NGO and Think Thank: Info on the Web for Business; November 4 : Other Treasures in the Collection for Business Needs. (p. 15)

Ces exemples ne sont qu'un infime reflet de la diversité des actions proposées par la Bibliothèque publique de Regina pour rejoindre les intérêts de ses diverses clientèles, mais ce choix, même si limité, illustre bien nos propos.

\section{CONCLUSION}

En bout de ligne, que ce soit : «Un dimanche aprèsmidi à la bibliothèque» ou «Une publication qui n'a pas son pareil», le message est le même: Une bibliothèque publique ou une bibliothèque publique comme 
lieu citoyen est effectivement un lieu ouvert à tous et où monsieur, madame et mademoiselle, à un moment donné, viendront puiser une parcelle de savoir, un moment de détente, une source d'inspiration.

À notre avis - on n'apprend pas que dans les livres -, si, comme l'affirme le Manifeste de l'UNESCO sur la bibliothèque publique, la bibliothèque publique est la porte locale d'accès à la connaissance, alors, de grâce, soyons attentifs à tous nos citoyens et gardons à l'esprit qu'il est tout à fait possible que l'on n'apprenne pas que dans les livres, que ces livres soient en format papier ou électronique.

En plus d'être attentifs à tous nos citoyens, et afin de faciliter ce passage obligé d'une institution culturelle à une institution démocratique, tel que décrit par Silvie Delorme, il serait grand temps d'ajuster notre vision de la bibliothèque publique. Pour ce faire, il ne faudrait peut-être plus adhérer sans discussion aux propos de certains de nos leaders :

Les citoyens eux-mêmes tendent à transformer les lieux en supermarché de la documentation : ils viennent y lire les journaux et périodiques, $y$ apprendre une langue, $y$ chercher de la documentation touristique, y naviguer sur Internet et souvent y correspondre, s'informer sur les lois et règlements de la cité ou de l'État, consulter annuaires et répertoires constamment mis à jour. C'est ainsi que les bibliothèques... risquent de se retrouver bourrées d'instruction mais légères de culture. Et cela alors que l'accès à la culture a, depuis les siècles des siècles où elles sont nées, été leur raison dêtre.
Mais, comme le souligne si bien Silvie Delorme (p. 46) :

En regard des risques impartis au rôle démocratique de la bibliothèque, le rôle culturel prend une coloration plus neutre. Tout aussi difficile à défendre, car la culture ne mobilise pas les foules, mais certainement plus prestigieux et plus sécuritaire pour les professionnels.

Dans un tel contexte, la bibliothèque publique comme lieu citoyen, ce n'est vraisemblablement pas pour demain.

\section{SOURCES CONSULTÉES}

@ the Library. Public Relations, Regina Public Library, Regina, Saskatchewan. Vol. 4, $\mathrm{n}^{\circ}$ 4, (Septembre/Octobre) 2004. $31 \mathrm{p}$.

Chapter E Verse. Fraser-Hickson Public Library, Montréal, Québec. Été 2004. 8.p.

Delorme, Silvie (200o). D'une institution culturelle à une institution démocratique. Passage obligé - L'ouverture citoyenne des bibliothèques publiques du Québec. Bulletin des bibliothèques de France, $\mathrm{n}^{\circ} 45$ (5), p. 42-46.

Le Grand Dictionnaire terminologique. Office de la langue française du Québec. http://www.granddictionnaire.com (consulté le 22 octobre 2004).

Joliceur, Louise (1998). Les groupes d'amis des bibliothèques publiques aux États-Unis et au Canada. Documentation et bibliothèques, $\mathrm{n}^{\circ} 44$ (3), p.121-128.

Le Robert illustré d'aujourd'hui. Paris, France Loisirs, 2000

UNESCO. Web World. Manifeste de l'Unesco sur la bibliothèque publique. http://www.unesco.org/webworld/libraries/ manifestos/libraman_fr.html (consulté le 22 octobre 2004).

Lise Bissonnette telle que citée dans Delorme, p. 45

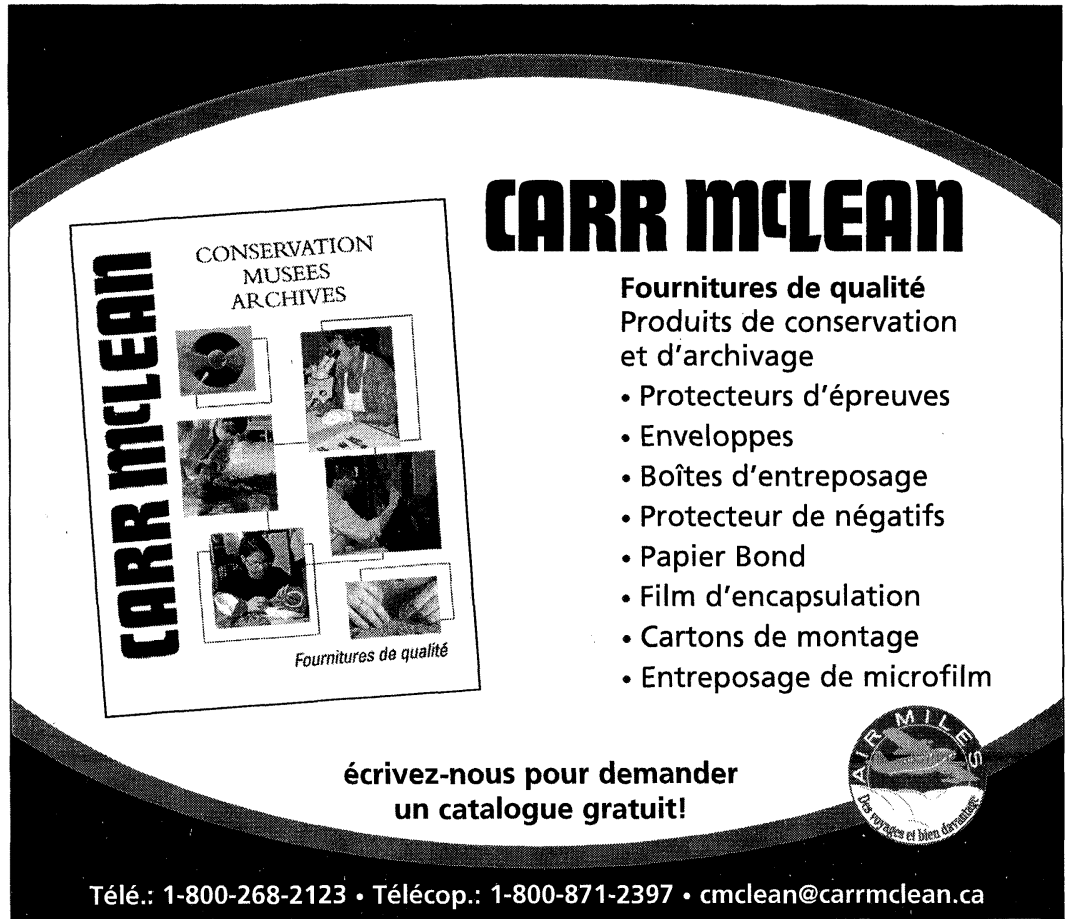

